\title{
The Impact of Having a 15-min Break With and Without Consuming an Energy Drink on Prolonged Simulated Highway Driving
}

\author{
Joris C. Verster ${ }^{1,2,3}$ • Aurora J. A. E. van de Loo ${ }^{1,2}$ - Adriana C. Bervoets ${ }^{2}$. \\ Loes Mooren ${ }^{2} \cdot$ Thomas Roth $^{4}$
}

Received: 18 July 2017 / Revised: 1 September 2017 / Accepted: 14 September 2017 / Published online: 21 September 2017

(C) The Author(s) 2017. This article is an open access publication

\begin{abstract}
Purpose To examine whether consuming an energy drink while having a break is effective in reducing the number of lapses of attention during prolonged highway driving.

Methods In a double-blind crossover study, $N=21$ healthy volunteers performed a 4-h driving test in the STISIM driving simulator. After $2 \mathrm{~h}$, a 15-min break was scheduled. During the break, participants consumed either $250 \mathrm{ml}$ of energy drink (ED) (Red Bull) or a placebo drink (Red Bull without caffeine ( $80 \mathrm{mg}$ ), glucuronolactone, taurine and B-vitamins). Participants were instructed to drive $95 \mathrm{~km} / \mathrm{h}$ with a steady lateral position. Primary outcome was the number (\#) of lapses; i.e., short periods of inattention defined by a deviation from the chosen lateral position for $>100 \mathrm{~cm}$ for $8 \mathrm{~s}$ or more.
\end{abstract}

Results Having a break, without consuming energy drink, significantly reduced the number of lapses in the 3rd hour of driving (9.9 versus 7.4 lapses, $p=0.006$ ). After placebo, the number of lapses in the 4th and 2nd hour were identical (9.9 and 9.9 lapses, $p=1.000$ ). Consuming and energy drink during the break resulted in a significant reduction in lapses in the 3rd hour (4.3 versus 9.2, $p=0.012$ ) and 4th hour of

Joris C. Verster

j.c.verster@uu.nl

1 Institute for Risk Assessment Sciences (IRAS), Utrecht University, Utrecht, The Netherlands

2 Division of Pharmacology, Utrecht University, Universiteitsweg 99, 3584 CG Utrecht, The Netherlands

3 Centre for Human Psychopharmacology, Swinburne University, Melbourne, Australia

4 Sleep Disorders and Research Center, Henry Ford Health System, Detroit, MI, USA driving (6.2 versus 9.2 lapses, $p=0.041$ ), when compared with the 2 nd hour of driving.

Conclusion Consuming an energy drink while having a 15-min break significantly reduces the number of lapses during prolonged highway driving, and to a greater extent than having a break only.

Keywords Driving $\cdot$ Accidents $\cdot$ Energy drink $\cdot$ Caffeine $\cdot$ Break

\section{Introduction}

The AAA Foundation for Traffic Safety showed that an estimated 21\% (6.400) of crashes in the USA involved a sleepy driver or a driver that had fallen asleep behind the wheel [1]. Driver sleepiness thus contributes to a significant number of traffic accidents, and continuous campaigns around the world warn for drowsy driving and point drivers to potential countermeasures to prevent being sleepy behind the wheel.

The effectiveness of several countermeasures for driver sleepiness has been scientifically investigated, including having a break, napping and the consumption of caffeinated beverages such as coffee or an energy drink. In particular, the effectiveness of caffeine to combat driver sleepiness and increase alertness has been the subject of many studies. For example, Horne and Reyner [2] examined the effects of caffeine on driving in sleep-deprived subjects. They drove for $30 \mathrm{~min}$ in a simulator, were given a placebo or a glucosebased energy drink (containing $160 \mathrm{mg}$ caffeine) and drove for another $2 \mathrm{~h}$. Thirty minutes after consumption of the energy drink, there were fewer driving incidents and reaction times were significantly improved when compared with the placebo condition. In a subsequent study applying a similar design, Reyner and Horne [3] confirmed that consumption 
of $250 \mathrm{ml}$ of energy drink containing $80 \mathrm{mg}$ caffeine significantly reduced the number of driving incidents. Subjective sleepiness was also significantly reduced. Several other studies also showed the positive effects of caffeinated beverages on driving performance in sleep-deprived drivers.

The current gold standard for outcome measure of driving tests is the standard deviation of lateral position (SDLP). SDLP, i.e., the weaving of the car, is an excellent outcome measure capable to demonstrate drug-induced impairment and dose-response effects, and has been applied successfully in drugs and driving research over the past 35 years [4, 5]. Using SDLP as an outcome measure, the effectiveness of caffeine has been demonstrated previously. For example, Mets et al. [6] examined the effects of energy drink $(250 \mathrm{ml}$ of Red Bull, containing $80 \mathrm{mg}$ caffeine) on prolonged simulated highway driving. Subjects had to drive for $2 \mathrm{~h}$ in the simulator, had a 15-min break in which they consumed energy drink or a placebo drink, followed by another $2 \mathrm{~h}$ of driving. In a subsequent study with the same design, Mets et al. [7] examined the effects of coffee ( $80 \mathrm{mg}$ caffeine) versus decaffeinated coffee. In both studies, in the caffeine condition, driving significantly improved in the $3 \mathrm{rd}$ and 4 th hour (significantly reduced SDLP values when compared to the placebo condition), and driver sleepiness was significantly reduced.

In real life, it has been demonstrated that the consumption of caffeinated substances significantly reduces the chances of having traffic accidents. For example, Sharwood et al. [8] showed that Australian long-distance truck drivers who consumed caffeinated substances had a $63 \%$ reduced likelihood of having a traffic accident when compared to drivers that did not consume caffeinated substances.

Examining driving accident risk in an experimental setting (e.g., driving simulator) is troublesome, as under normal driving circumstances having an accident is a rare event. Thus, measuring accidents is not a useful outcome measure on driving tests. The relationship between SDLP and the risk of having an accident is unclear, although indirect evidence suggests such a relationship exists [9]. Therefore, the search for other proxy measures of accidents has continued. One of the proposed assays is number of lapses (i.e., periods of microsleep). It is assumed that during these short periods of inattention, the risk of having accidents is significantly increased. A lapse is a short period of inattention and is defined as a deviation from the mean lateral position for $>100 \mathrm{~cm}$ for $8 \mathrm{~s}$ or more [10]. An example of a lapse is given in Fig. 1.

In previous analyses, we showed that in healthy volunteers after intake of hypnotic drugs known to have residual effects, the number of lapses during a next-morning drive were significantly increased compared to a normal drug-free night of sleep [10]. Alternatively, in patients with attention deficit hyperactivity disorder (ADHD) the number of lapses

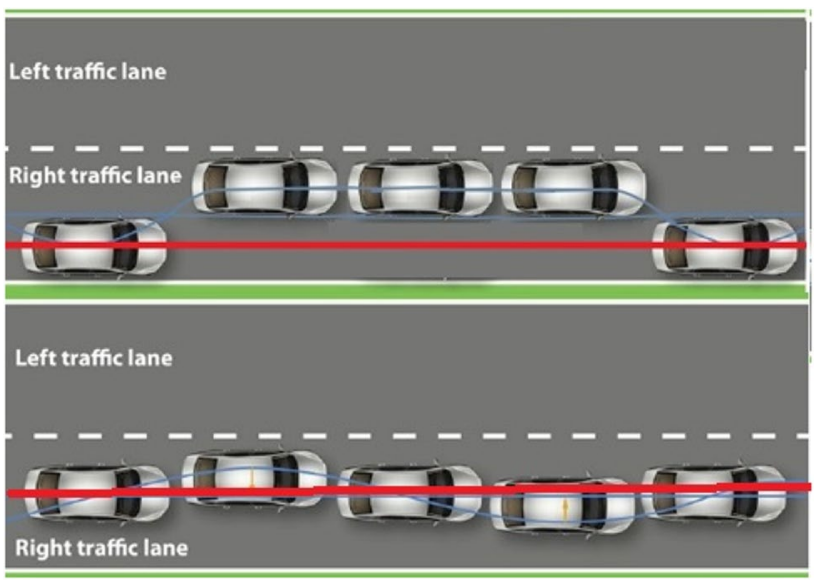

Fig. 1 Schematic representation of a lapse. Subjects were instructed to drive with a steady later position within the right traffic lane (see bottom figure). A lapse occurs if drivers deviate more than $100 \mathrm{~cm}$ from their chosen position for more than $8 \mathrm{~s}$ (see top figure)

significantly reduced after treatment with methylphenidate [11].

It is important to demonstrate the efficacy of alertnessenhancing compounds using tests with relevance to real life activities which are compromised by sleepiness, such as prolonged highway driving. For this purpose, lapses are an ideal outcome measure. Therefore, the aim of the current study was to examine whether consumption of an energy drink while having a break has an effect of the number of lapses during prolonged simulated highway driving.

\section{Methods}

Data from Mets et al. [6] were re-analyzed. Twenty-four healthy volunteers participated in this double-blind, randomized, placebo-controlled, crossover study. Subjects were included if they were healthy non-smokers, were moderate caffeine consumers (two to four glasses of caffeine-containing beverages per day, infrequent energy drink consumers ( $<1$ drink per month), and had regular sleeping hours, aged 21-35 years old. Subjects were included if they had a valid driver's license for at least 3 years and drive a minimum of $5000 \mathrm{~km}$ per year. Subjects with sleep disorders or an Epworth Sleepiness Scale score above 10 were excluded from participation. Details on recruitment and inclusion and exclusion criteria are described by Mets et al. [6]. No formal ethical approval was required according to the Medical Ethical Committee of the University Medical Center Utrecht for the present reanalysis. Ethics permission was obtained for the original two studies from the Medical Ethical Committee of the University Medical Center Utrecht. Written informed 
consent was obtained from each subject before taking part in the study.

The study comprised a training session, and two test days. On each test day, subjects completed a 4-h simulated highway driving test, aiming to mimic the on-road driving test in actual traffic [12]. Driving tests were performed using STISIM Drive ${ }^{\mathrm{TM}}$ (version M300, Systems Technology, Inc., Hawthorne, CA, USA). To avoid practice effects, several variants of a $100-\mathrm{km}$ highway driving test scenario, developed by EyeOctopus B.V., were used for this study.

Subjects were instructed to drive with a steady lateral position within the right lane while maintaining a constant speed of $95 \mathrm{~km} / \mathrm{h}$ [4]. The primary outcome measure for the current analyses was the number of lapses.

After $2 \mathrm{~h}$ of driving, a 15 -min break was scheduled. During the break, subjects consumed either $250 \mathrm{ml}$ of energy drink (Red Bull, containing $21 \mathrm{~g}$ of sucrose, $5 \mathrm{~g}$ of glucose, $1 \mathrm{~g}$ of taurine, $80 \mathrm{mg}$ of caffeine, $60 \mathrm{mg}$ of glucuronolactone, $50 \mathrm{mg}$ of inositol, and B vitamins) or a placebo energy drink ( $250 \mathrm{ml}$ of Red Bull without caffeine, glucuronolactone, taurine, inositol and B-vitamins). The samples were provided by Red Bull GmbH. The latter was important to assure treatment blinding with regard to color and taste. The 15-min break was followed by another $2 \mathrm{~h}$ of driving.

Subjects were tested either in the morning (8:00-13:00 h) or afternoon (13:00-17:00 h) in a balanced manner and started each test day at the same time. Test days were separated by a wash-out period of at least 3 days.

Statistical analyses were performed using SPSS, version 24. For each subject, and each condition, the number of lapses were calculated for each hour of driving. For each hour of driving, the non-parametric related samples Wilcoxon signed-rank test was used to compare the number of lapses after consuming energy drink or placebo, and between the different hours of driving. Differences were considered significant if $p<0.05$.

\section{Results}

Twenty-four subjects participated in the study. Three subjects were excluded due to protocol violations. Data from twenty-one subjects (nine men and twelve women) were used for the statistical analysis. Their mean (SD) age was 22.8 (1.4) years old.

Mean (SD) number of lapses after placebo and energy drink, and descriptive data are summarized in Table 1. The number of lapses after placebo and energy drink are visualized in Fig. 2.

No significant differences were observed between the energy drink and placebo in the number of lapses observed in hour $1(p=1.00)$ and in hour $2(p=0.600)$.

Having a break, without consuming energy drink, significantly reduced the number of lapses in the third hour of driving when compared with the second hour of driving $(p=0.006)$. However, the number of lapses in the second hour of driving after placebo was identical $(p=1.000)$. When compared to the second hour of driving, consuming an energy drink during the break resulted in a significant reduction in lapses in the third hour $(p=0,012)$ and the fouth hour of driving ( $p=0.041$ ).

When directly comparing the two treatments, in hour 3 , fewer lapses were observed after consuming energy drink when compared to the placebo drink, although the difference

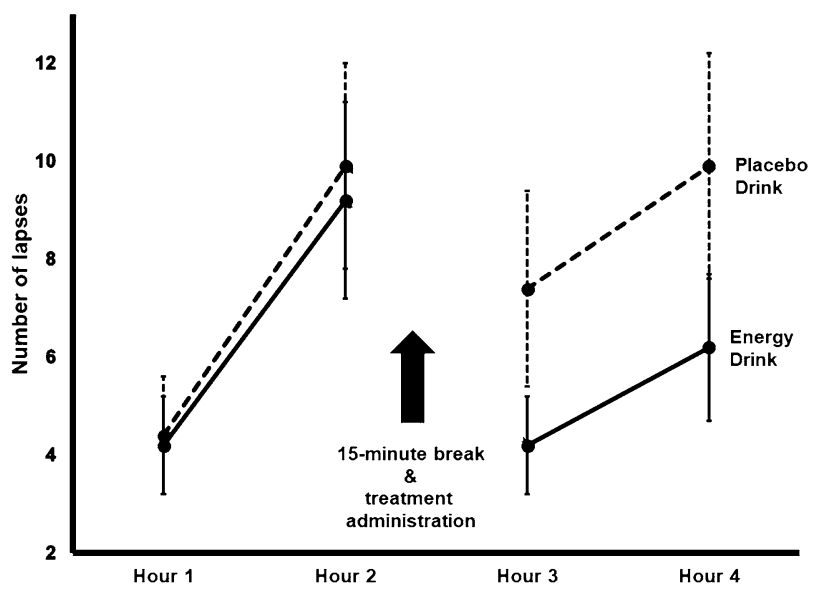

Fig. 2 Number of lapses. Means and standard errors are shown. Compared to the second hour, after consuming energy drink, the number of lapses was significantly reduced in the third and fourth hour of driving. After placebo, the difference with the second hour was significant in the third hour of driving. However, in the fourth hour after placebo, the number of lapses were identical to those observed in the second hour
Table 1 Number of lapses: descriptives

\begin{tabular}{|c|c|c|c|c|c|c|c|c|}
\hline & \multicolumn{4}{|c|}{ Energy drink } & \multicolumn{4}{|c|}{ Placebo } \\
\hline & Mean & $\mathrm{SD}$ & Median & $\overline{\mathrm{IQR}}$ & $\overline{\text { Mean }}$ & SD & Median & IQR \\
\hline Hour 1 & 4.3 & 4.7 & 3.0 & 4.5 & 4.4 & 5.5 & 3.0 & 4.5 \\
\hline Hour 2 & 9.2 & 9.4 & 6.0 & 11.0 & 9.9 & 9.6 & 7.0 & 11.0 \\
\hline Hour 3 & 4.3 & 4.3 & 4.0 & 4.5 & 7.4 & 9.2 & 4.0 & 8.0 \\
\hline Hour 4 & 6.2 & 6.9 & 4.0 & 7.0 & 9.9 & 10.5 & 7.0 & 10.0 \\
\hline
\end{tabular}

$S D$ standard deviation, $I Q R$ inter-quartile range 
did not reach statistical significance $(p=0.129)$. In hour 4 , significantly fewer lapses were observed after consuming energy drink when compared to the placebo drink $(p=0.024)$.

\section{Discussion}

This study showed that having a 15-min break in combination with consuming an energy drink significantly reduces the number of lapses during prolonged simulated highway driving. Thus, the consumption of energy drink showed to be an effective countermeasure for drowsy driving in nonsleep-deprived healthy subjects.

The number of lapses in the second hour of driving and fourth hour of driving after placebo was identical, suggesting that after $2 \mathrm{~h}$ of driving having a 15-min break is recommended per se.

Our findings are in line with the original analyses by Mets et al. [6], using SDLP as outcome measure. After consuming energy drink, SDLP was significantly reduced in both hour 3 and 4 . In the current analyses, a significant reduction in the number of lapses was also seen in hour 3 and hour 4 . In addition, the findings are in accordance with the significant reduction in driver sleepiness observed after consuming energy drink [6]. Our study confirms that the number of lapses increases over distance driven, and that having a break is an effective way to counteract drowsy driving and reduce the number of lapses. In combination with consuming an energy drink during the break, the reduction in lapses is significantly increased, illustrating the effectiveness of caffeinated beverages as countermeasure for drowsy driving.

A limitation of this study is the fact that we used a driving simulator and not tested the effects on a public highway in actual traffic. It is known that drivers may perform differently in a driving simulator, as no actual risks are involved [5]. This may also be reflected in reduced motivation to perform the test. Driving simulators also showed to be less sensitive in detecting relative modest drug effects when directly compared to on-road driving [13]. Further, sleepiness scores are usually higher in simulator settings when compared to on-road driving tests [14]. Future research should replicate these findings on-road in actual traffic. It would be interesting to record EEG activity during these driving tests and link these observations to the occurrence of lapses. This way it can be demonstrated that lapses are indeed short periods of inattention (i.e., microsleep) and how they directly relate to the driver's performance.

In conclusion, consuming energy drink while having a break significantly reduces the number of lapses during prolonged simulated highway driving. Our findings support previous evidence that the consumption of caffeinated beverages contributes to a significant reduction in the likelihood of having traffic accidents.

\section{Compliance with ethical standards}

Funding This study was funded by Red Bull GmbH and Utrecht University. Red Bull $\mathrm{GmbH}$ was not involved in the design and conduct of the study, collection, management, analysis, interpretation of the data, or preparation of the manuscript.

Conflict of interest Joris Verster has received Grants/research support from the Dutch Ministry of Infrastructure and the Environment, Janssen Research and Development, Nutricia, Red Bull, Sequential, and Takeda, and has acted as a consultant for the Canadian Beverage Association, Centraal Bureau Drogisterijbedrijven, Coleman Frost, Danone, Deenox, Eisai, Janssen, Jazz, Purdue, Red Bull, Sanofi-Aventis, Sen-Jam Pharmaceutical, Sepracor, Takeda, Transcept, Trimbos Institute, and Vital Beverages. Thomas Roth has received Grants/research support from Aventis, Cephalon, GlaxoSmithKline, Neurocrine, Pfizer, Sanofi, Schering-Plough, Sepracor, Somaxon, Syrex, Takeda, TransOral, Wyeth and Xenoport; has acted as a consultant for Abbott, Acadia, Acoglix, Actelion, Alchemers, Alza, Ancil, Arena, AstraZeneca, Aventis, AVER, BMS, BTG, Cephalon, Cypress, Dove, Elan, Eli Lilly, Evotec, Forest, GlaxoSmithKline, Hypnion, Impax, Intec, Intra-Cellular, Jazz, Johnson \& Johnson, King, Lundbeck, McNeil, MediciNova, Merck, Neurim, Neurocrine, Neurogen, Novartis, Orexo, Organon, Prestwick, ProctereGamble, Pfizer, Purdue, Resteva, Roche, Sanofi, Schering-Plough, Sepracor, Servier, Shire, Somaxon, Syrex, Takeda, TransOral, Vanda, Vivometrics, Wyeth, Yamanuchi, and Xenoport. The other authors have no conflicts of interest to disclose.

Open Access This article is distributed under the terms of the Creative Commons Attribution 4.0 International License (http://creativecommons.org/licenses/by/4.0/), which permits unrestricted use, distribution, and reproduction in any medium, provided you give appropriate credit to the original author(s) and the source, provide a link to the Creative Commons license, and indicate if changes were made.

\section{References}

1. Tefft BC. Prevalence of motor vehicle crashes involving drowsy drivers, United States, 2009-2013. AAA Foundation for Traffic Safety. 2014. https://www.aaafoundation.org/sites/default/files/ AAAFoundation-DrowsyDriving-Nov2014.pdf. Accessed 17 July 2017.

2. Horne JA, Reyner LA. Beneficial effects of an "energy drink" given to sleepy drivers. Amino Acids. 2001;20:83-9.

3. Reyner LA, Horne JA. Efficacy of a 'functional energy drink' in counteracting driver sleepiness. Physiol Behav. 2002;75:331-5.

4. Verster JC, Roth T. Standard operation procedures for conducting the on-the-road driving test, and measurement of the standard deviation of lateral position (SDLP). Int J Gen Med. 2011;4:359-71.

5. Verster JC, Roth T. Thirty years of Dutch on-the-road driving research: strengths and limitations of the highway driving test and future challenges. Curr Psychopharmacol. 2012;1:97-102.

6. Mets MAJ, Ketser S, Blom C, van Gerven M, van Willigenburg GM, Olivier B, Verster JC. Positive effects of Red Bull ${ }^{\circledR}$ Energy Drink on driving performance during prolonged driving. Psychopharmacology. 2011;214:737-44. 
7. Mets MAJ, Baas D, van Boven I, Olivier B. Effects of caffeine versus placebo on prolonged highway driving. Psychopharmacology. 2012;222:337-42.

8. Sharwood LN, Elkington J, Meuleners L, Ivers R, Boufous S, Stevenson M. Use of caffeinated substances and risk of crashes in long distance drivers of commercial vehicles: case-control study. BMJ. 2013;346:f1140.

9. Owens K, Ramaekers JG. Drugs, driving, and models to measure driving impairment. In: Verster JC, Pandi-Perumal SR, Ramaekers JG, de Gier JJ, editors. Drugs, driving and traffic safety. Basel: Birkhauser Verlag AG; 2009. p. 43-58.

10. Verster JC, Bervoets AC, de Klerk S, Roth T. Lapses of attention as outcome measure of the on-the-road driving test. Psychopharmacology. 2014;231:283-92.

11. Verster JC, Roth T. Methylphenidate significantly reduces lapses of attention during on-road highway driving in patients with ADHD. J Clin Psychopharmacol. 2014;34:663-6.
12. Mets MAJ, Kuipers E, de Senerpont Domis LM, Leenders M, Volkerts ER, Olivier B, Verster JC. Effects of alcohol highway driving in the STISIM driving simulator. Hum Psychopharmacol. 2011;26:434-9.

13. Veldstra JL, Bosker WM, de Waard D, Ramaekers JG, Brookhuis KA. Comparing treatment effects of oral THC on simulated and on-the-road driving performance: testing the validity of driving simulator drug research. Psychopharmacology. 2015;232:2911-9.

14. Helland A, Jenssen GD, Lervåg LE, Moen T, Engen T, Lydersen S, Mørland J, Slørdal L. Evaluation of measures of impairment in real and simulated driving: results from a randomized, placebocontrolled study. Traffic Inj Prev. 2016;17:245-50. 